\title{
Rule Out Normal Tension Glaucoma in a Young Woman
}

Jonathan C Chou ${ }^{1}$, Robert M Mallery ${ }^{1,2}$ and Louis R Pasquale ${ }^{1,3^{*}}$

${ }^{1}$ Massachusetts Eye and Ear, Department of Ophthalmology, Harvard Medical School, Boston, MA, USA

${ }^{2}$ Department of Neurology, Brigham and Women's Hospital, Harvard Medical School, Boston, MA, USA

${ }^{3}$ Channing Division of Network Medicine, Brigham and Women's Hospital, Boston, MA, USA

"Corresponding author: Louis R Pasquale, Massachusetts Eye and Ear, Department of Ophthalmology, Harvard Medical School, Boston, MA, USA, Tel: 3124348310; Fax: 3124348310; E-mail: jchou87@gmail.com

Received date: December 21, 2017; Accepted date: January 2, 2018; Published date: January 7, 2018

Copyright: $\odot 2018$ Chou JC, et al. This is an open-access article distributed under the terms of the Creative Commons Attribution License, which permits unrestricted use, distribution, and reproduction in any medium, provided the original author and source are credited.

Keywords: Glaucoma; Optic neuropathy; Superior segmental optic nerve hypoplasia

\section{Case Report}

A 44 year-old Caucasian woman presented with one year of difficulty driving at night. She perceived an intermittent film over the inferior part of her vision in both eyes that was worse in the dark. An outside ophthalmologist noted bilateral inferior arcuate defects on visual fields (Figure 1) and referred her for possible glaucoma.

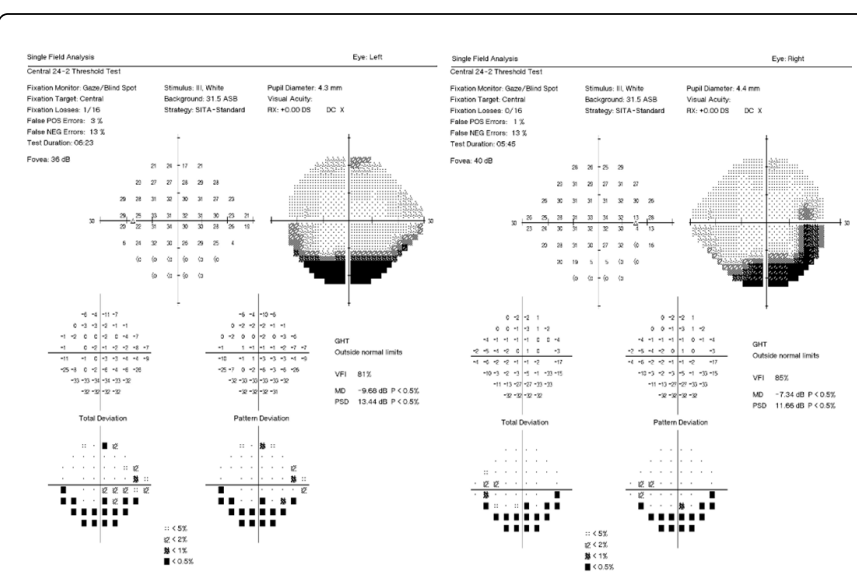

Figure 1: 24-2 Humphrey Visual Fields of the left and right eye, respectively. There is a deep inferior arcuate depression in each eye.

There was no significant ocular history. Her medical history was remarkable for pulmonary lymphangioleiomyomatosis (LAM) and obstructive sleep apnea. Medications included leuprorelin and fulvestrant, both anti-estrogen drugs used in the treatment of LAM. The patient's mother had type 1 diabetes mellitus, and her maternal aunt had an optic neuropathy of unknown cause.

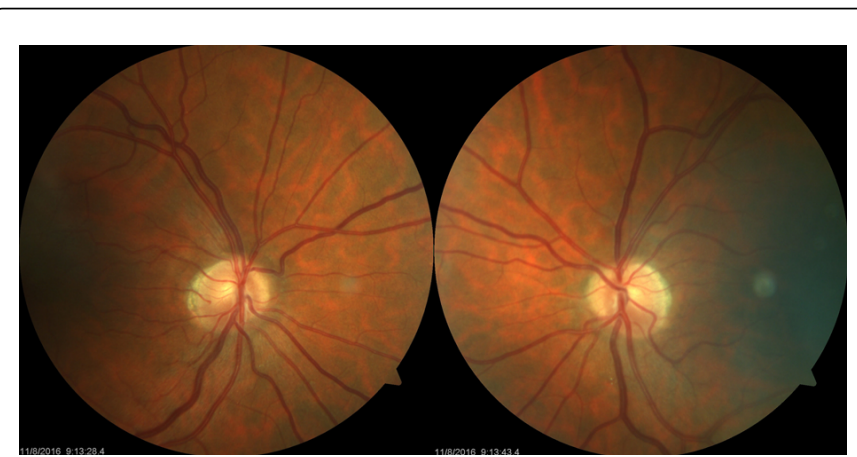

Figure 2: Color Fundus Photography of the right and left optic discs, respectively. The optic nerves appear hypoplastic with a scleral ring superonasally and temporally ("double-ring" sign). The central retinal artery has a more superonasal entrance onto the optic nerve due to the hypoplasia of the superior optic disc tissue.

Examination showed best-corrected visual acuities of 20/20-1 OU, intraocular pressures of $16 \mathrm{~mm} \mathrm{Hg} \mathrm{OU}$, normally reactive pupils, and no dyschromatopsia. Central corneal thicknesses were 560 um bilaterally, and gonioscopy revealed open angles without increased pigmentation. Her anterior segment exam was unremarkable. Fundus examination revealed bilateral hypoplastic optic nerves greatest superiorly without enlarged cup to disc ratios (Figure 2). Optical coherence tomography (OCT) confirmed hypoplastic nerves with small cup to disc ratios and thinning of the retinal nerve fiber layer (RNFL) superiorly (Figure 3). Further workup revealed normal MRI of the orbits with and without contrast, and normal full field and multifocal ERG. 


\begin{tabular}{|r|c|c|}
\hline \multicolumn{1}{|c|}{ \ } & OD & OS \\
\hline Average RNFL Thickness & $66 \mu \mathrm{m}$ & $64 \mu \mathrm{m}$ \\
\hline RNFL Symmetry & \multicolumn{2}{|c|}{$96 \%$} \\
\hline Rim Area & $1.09 \mathrm{~mm}^{2}$ & $1.12 \mathrm{~mm}^{2}$ \\
\hline Disc Area & $1.13 \mathrm{~mm}^{2}$ & $1.13 \mathrm{~mm}^{2}$ \\
\hline Average C/D Ratio & 0.20 & 0.12 \\
\hline Vertical C/D Ratio & 0.26 & 0.12 \\
\hline Cup Volume & $0.006 \mathrm{~mm}^{3}$ & $0.002 \mathrm{~mm}^{3}$ \\
\hline
\end{tabular}

RNFL Thickness
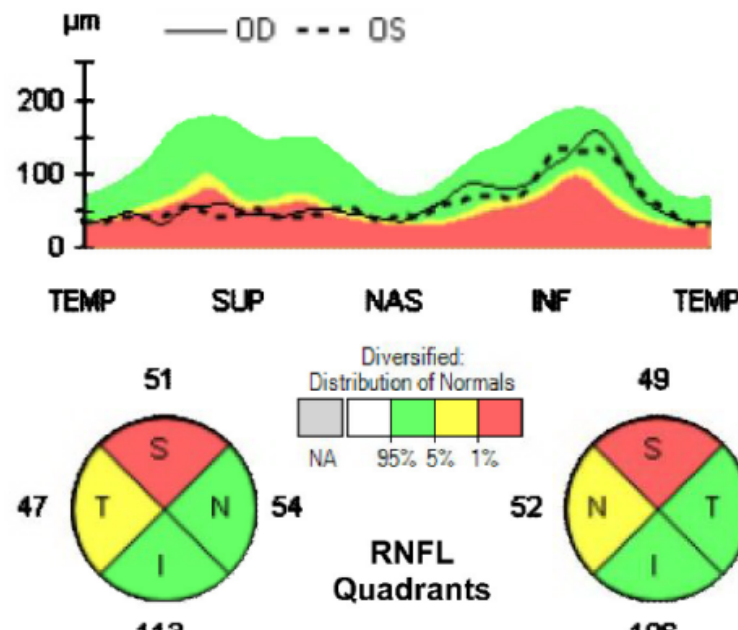

Diversified:

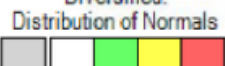
NA $95 \% 5 \%, 1 \%$ 54

RNFL Quadrants

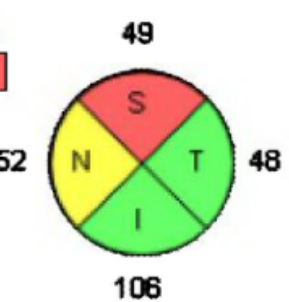

113

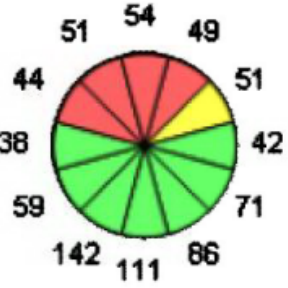

RNFL

Clock

Hours

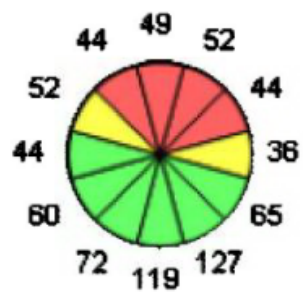

Figure 3: Optic nerve head and retinal nerve fiber layer analysis of the right and left eyes, respectively. Optic nerve analysis reveals hypoplastic nerves $\left(1.13 \mathrm{~mm}^{2}\right)$ with small cup to disc ratio. Retinal nerve fiber layer analysis shows significant thinning superiorly bilaterally.

Upon further questioning, it was revealed that the patient's halfsister (same mother) also had an optic neuropathy with inferior visual field loss in the right eye. Examination of the sister showed superior optic nerve hypoplasia, more significantly affecting the right eye (Figure 4).

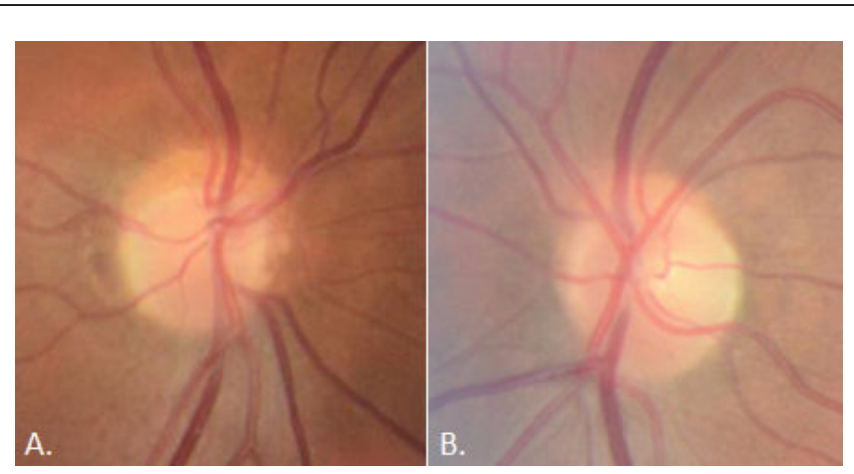

Figure 4: Optic disc photos of patient's sister. The nerves are hypoplastic superiorly with associated superior scleral rings, right greater than left (A and B). There is absence of discernible superior nerve tissue OD (A).

Our patient was subsequently diagnosed with superior segmental optic nerve hypoplasia.

\section{Discussion}

Patients with optic nerve hypoplasia can have visual acuity ranging from 20/15 to light perception. The majority of patients have bilateral involvement. Close examination of the optic nerves typically reveal a "double-ring" sign where the inner ring corresponds to the hypoplastic nerve and the outer ring corresponds to the junction between the sclera and lamina cribrosa [1]. Superior segmental optic nerve hypoplasia $(\mathrm{SSOH})$ is a subcategory of optic nerve hypoplasia and is also known as "topless disc syndrome." It is a congenital anomaly that primarily affects the superior optic nerve and retinal nerve fiber layer with a corresponding inferior visual field defect. It has a strong association with maternal diabetes, and one study also showed a strong association with paternal ischemic heart disease [2]. The exact pathophysiology is unknown, but it is hypothesized that the disorder is due to a primary failure of retinal ganglion cell (RGC) development and/or exaggerated apoptosis of RGCs due to fetal vascular insult $[3,4]$.

SSOH can resemble normal tension glaucoma due to the similar visual field defects, RNFL thinning, and normal intraocular pressures; however, there are several ways of differentiating the two entities. Patients with $\mathrm{SSOH}$ have smaller overall disc areas and usually have a normal cup to disc ratio. Using OCT, patients with $\mathrm{SSOH}$ have a thinner RNFL superiorly, but a thicker ganglion cell layer-inner plexiform layer (GCL-IPL) [5]. Furthermore, thinning and posterior displacement of the lamina cribrosa are distinctive features of glaucoma that are not seen in $\mathrm{SSOH}$ [6]. SSOH is non-progressive, and no treatment is necessary. There have been case reports of $\mathrm{SSOH}$ that is also accompanied by NTG with progressive glaucomatous visual field defect [7].

This case was unusual as she did not become aware of her symptoms until her early 40s while undergoing treatment of LAM. Pulmonary LAM is a rare lung disease where the smooth muscle lining of lung parenchyma and blood vessels proliferate abnormally. As it tends to affect women of childbearing age and worsens during pregnancy, antiestrogen therapy is a treatment, although its use is controversial $[8,9]$. Our patient was on two anti-estrogen medications, leuprorelin, a gonadotropin releasing hormone analog designed to reduce serum estradiol levels, and fulvestrant, an estrogen receptor antagonist. It has 
been shown that the optic nerve has receptors for estradiol and that estrogen deficiency can accelerate aging of the optic nerve [10,11]. To our knowledge, there have been no reports of glaucoma and LAM. However, fulvestrant has been reported to block the neuroprotective effect of estradiol in an animal model of glaucoma [12]. While the reason for this patient's symptoms of visual disability is unclear, and they could be related to acute onset of awareness of a stationary visual disability, we did entertain the possibility that her visual symptoms were exacerbated by anti-estrogen therapy.

This case was challenging due to the late presentation of the patient. However, the patient's exam, OCT, visual fields, as well as her sister's presentation from the same mother with type I diabetes helped to make the diagnosis.

\section{Conclusion}

In conclusion, $\mathrm{SSOH}$ is a congenital optic nerve anomaly. The exam shows a non-progressive hypoplasia of the superior optic disc tissue and superior RNFL thinning on OCT, with corresponding inferior visual field loss. Distinctive features of the optic nerve and OCT help differentiate it from glaucoma. Follow-up for these patients is recommended to ensure that there is no progressive visual field loss to suggest a superimposed glaucoma.

\section{Informed Consent}

Not obtained. Personal identifying information was removed from this report as informed consent to publish such information was not obtained.

\section{References}

1. Pilat A, Sibley D, McLean RJ, Proudlock FA, Gottlob I (2015) HighResolution Imaging of the Optic Nerve and Retina in Optic Nerve Hypoplasia. Ophthalmol 122: 1330-1339.
2. Seo S, Lee CE, Kim DW, Kim YK, Jeoung JW, et al. (2014) Prevalence and risk factors of superior segmental optic hypoplasia in a Korean population: the Korea National Health and Nutrition Examination Survey. BMC Ophthalmol 14: 157.

3. Kim RY, Hoyt WF, Lessell S, Narahara MH (1989) Superior segmental optic hypoplasia. A sign of maternal diabetes. Arch Ophthalmol 107: 1312-1315.

4. Landau K, Bajka JD, Kirchschlager BM (1998) Topless optic disks in children of mothers with type I diabetes mellitus. Am J Ophthalmol 125: 605-611.

5. Han JC, Choi DY, Kee C (2015) The Different Characteristics of Cirrus Optical Coherence Tomography between Superior Segmental Optic Hypoplasia and Normal Tension Glaucoma with Superior Retinal Nerve Fiber Defect. J Ophthalmol 2015: 641204.

6. Lee EJ, Lee KM, Lee SH, Kim TW (2016) Comparison of the Deep Optic Nerve Structures in Superior Segmental Optic Nerve Hypoplasia and Primary Open-Angle Glaucoma. J Glaucoma 25: 648-656.

7. Yamazaki Y, Hayamizu F (2012) Superior segmental optic nerve hypoplasia accompanied by progressive normal-tension glaucoma. Clin Ophthalmol 6: 1713-1716.

8. Glasgow CG, Steagall WK, Taveira-Dasilva A, Pacheco-Rodriguez G, Cai $\mathrm{X}$, et al. (2010) Lymphangioleiomyomatosis (LAM): molecular insights lead to targeted therapies. Respir Med 1: S45-58.

9. Harknett EC, Chang WY, Byrnes S, Johnson J, Lazor R, et al. Use of variability in national and regional data to estimate the prevalence of lymphangioleiomyomatosis. QJM 104: 971-979.

10. Munaut C, Lambert V, Noel A, Frankennea F, Deprez M, et al. (2001) Presence of oestrogen receptor type beta in human retina. $\mathrm{Br} \mathrm{J}$ Ophthalmol 85: 877-882.

11. Vajaranant TS, Pasquale LR (2012) Estrogen deficiency accelerates aging of the optic nerve. Menopause 19: 942-947.

12. Russo R, Cavaliere F, Watanabe C, Nucci C, Bagetta G, et al. (2008) 17Beta-estradiol prevents retinal ganglion cell loss induced by acute rise of intraocular pressure in rat. Prog Brain Res 173: 583-590. 